\title{
Civilisations
}

Revue internationale d'anthropologie et de sciences

humaines

61-1| 2012

Au-delà du consensus patrimonial

\section{Nouveaux acteurs du patrimoine, nouvelles postures anthropologiques}

A propos de Ethnologues et passeurs de mémoires

\section{Chiara Bortolotto}

\section{(2) OpenEdition}

Journals

Édition électronique

URL : http://journals.openedition.org/civilisations/3118

DOI : $10.4000 /$ civilisations.3118

ISSN : 2032-0442

Éditeur

Institut de sociologie de l'Université Libre de Bruxelles

\section{Édition imprimée}

Date de publication : 22 décembre 2012

Pagination : 139-146

ISBN : 2-87263-038-4

ISSN : 0009-8140

Référence électronique

Chiara Bortolotto, « Nouveaux acteurs du patrimoine, nouvelles postures anthropologiques »,

Civilisations [En ligne], 61-1 | 2012, mis en ligne le 30 décembre 2015, consulté le 30 avril 2019. URL:

http://journals.openedition.org/civilisations/3118; DOI : 10.4000/civilisations.3118 


\title{
Nouveaux acteurs du patrimoine, nouvelles postures anthropologiques
}

\author{
À propos de Ethnologues et passeurs de mémoires
}

\section{Chiara BORTOLOTTO}

À l'heure où la participation des acteurs sociaux dans la mise en œuvre des politiques publiques est en train de s'instituer comme une norme globale, et où le traitement expert du patrimoine doit par conséquent négocier sa place avec de nouveaux acteurs, Ethnologues et passeurs de mémoires offre une outil de réflexion essentiel tant pour les anthropologues que pour les professionnels du patrimoine. Complexe et controversé, ce tournant participatif, par lequel l'Unesco aspire à mettre les communautés au cœur du processus de patrimonialisation, interroge en effet l'ensemble des acteurs du patrimoine. Cet ouvrage, dirigé par Gaetano Ciarcia, revêt cependant un intérêt particulier pour les acteurs institutionnels, désormais appelés à dialoguer directement avec les passeurs de mémoire et à repenser par conséquent les fondements de leur mission, par ailleurs considérée comme éminemment technique. La nouvelle démarche patrimoniale prônée par les instances internationales remet en fait en cause les critères et les acteurs qui président à l'attribution d'une valeur patrimoniale et se revendique explicitement comme un processus d'identification. L'impulsion identitaire et émotionnelle de la mise en patrimoine, fondée sur les valeurs et critères subjectifs de ses acteurs, est bien connue par les ethnologues et les historiens (Fabre 2002; Le Goff 1998 ; Poulot 2001). Sans compter que des patrimonialisations profanes, réalisées en parallèle, sinon en conflit avec les institutions patrimoniales et leurs critères, deviennent aujourd'hui une préoccupation concrète des agences culturelles, désormais appelées à les intégrer dans la fabrique autorisée du patrimoine. Or, dans ce processus d'institutionnalisation, les figures de « heritage bearers » sont constamment mobilisées. Une des ambitions déclarées en ouverture de cet ouvrage est celle de présenter une vision réaliste de ces acteurs, bien distincte de celle mise en avant par le discours des instances internationales, vision que Gaetano Ciarcia considère comme une image stéréotypée.

La pluralité des figures et les modalités de passation présentées dans ce volume, tout en permettant de comprendre la complexité de l'agencéité de ces acteurs, ont le mérite de monter leur singularité, souvent effacée dans les documents programmatiques et opérationnels des institutions. Ce volume présente et analyse les traits constitutifs des acteurs aujourd'hui chargés d'un devoir mémoriel, que ce soit dans la rencontre avec l'ethnologue sur le terrain ou dans le cadre de leur vie sociale. Non seulement cette analyse fait écho aux préoccupations qui animent le plus ample débat social, institutionnel et politique, mais elle se place au coeur de la réflexion scientifique 
contemporaine. Cet ouvrage témoigne en effet de l'intérêt que le thème de la transmission à l'ombre de la problématique patrimoniale représente pour l'ethnologie contemporaine (Berliner 2010 ; Adell et Pourcher 2011). Elle se distingue toutefois par le choix de saisir ce processus à travers les figures des médiateurs qui font la transmission. Le premier mérite de ce travail réside donc dans le choix d'un angle originel pour analyser les phénomènes de transmission et de mise en patrimoine. Nous allons cependant nous attarder sur un autre aspect intéressant de cet ouvrage, lui aussi central pour le débat anthropologique contemporain : la nouvelle relation que l'ethnologue entretient avec ses interlocuteurs face à leur transformation en agents autonomes du patrimoine.

\section{Passeurs de mémoires}

Le point de départ de cet ouvrage stimulant est le constat, clairement posé par Gaetano Ciarcia, d'une « transformation progressive de la figure du détenteur indigène de connaissances traditionnelles, et donc d'informations ethnographiques potentielles, en concepteur et passeur de matériaux culturels à patrimonialiser ». Cette transformation reste encadrée dans une relation ethnographique lorsque ces passeurs, bien que distincts des interlocuteurs « ordinaires », interagissent avec le chercheur. Dans certains cas, leur « capital discursif» est mis au profit de leurs intérêts particuliers dans une visée explicitement pragmatique, et le chercheur devient un instrument qui leur permet de participer au processus de patrimonialisation. Dans d'autres cas, cette transformation du « détenteur indigène » peut évoluer jusqu' à une autonomisation de son rôle lorsqu'il intervient à la première personne dans l'arène scientifique ou institutionnelle sans la médiation d'un ethnologue diplômé. Dans les deux cas, nous sommes face à l'émergence d'agents non institutionnels de la mise en patrimoine.

Qui sont ces passeurs ? Des « hommes-patrimoine», selon Nicolas Adell, capables non seulement de conserver des savoir-faire mais aussi de les faire évoluer. Ainsi, ce qui est transmis est à la fois des idées, des objets, des expériences et des valeurs. Si les membres de la communauté - dans le cas de l'étude d'Adell, celle des compagnons du tour de France - sont les responsables de la transmission des trois derniers domaines, il y a des passeurs qui ont avec la communauté un rapport d'extériorité et n'interagissent avec elle que dans des circonstances spécifiques : lorsqu'il s'agit de relayer les représentations de la culture communautaire vers l'extérieur, en direction du monde scientifique ou institutionnel. Les chercheurs, historiens ou ethnologues, deviennent alors des passeurs d'idées dont les membres de la communauté se saisissent pour en faire un usage instrumental. Cette « usabilité » des ethnologues peut mener à un véritable basculement des rôles : l'ethnologue est alors sollicité et mobilisé dans leurs propres entreprises patrimoniales par ceux qui ont traditionnellement été ses interlocuteurs sur le terrain. Véronique Dassié et Julie Garnier nous présentent plusieurs exemples de cette situation où la figure du chercheur est transformée en personne-ressource, «médiateur d'un projet de reconnaissance », dans les projets liées à la mémoire de l'immigration en France. Ici, la simple présence de l'ethnologuepasseur - plutôt que ses compétences professionnelles réelles - légitime la démarche patrimoniale. Les artifices menant à l'utilisation du chercheur sont exposés également par Nicolas Puig à propos d'une collecte d'objets associés au métier de musicien 
au Caire. Le chercheur devient ainsi une personne-ressource pour ses interlocuteurs qui, par la simple fréquentation d'un expert étranger, gagnent une aura de légitimité artistique.

Si l'intervention de chercheurs diplômés peut aider au passage des intérêts des acteurs sociaux dans des arènes lointaines de leur vie quotidienne, les chercheurs indigènes incarnent une forme différente de passeurs. Philippe Martel nous présente deux figures de dialectologues autodidactes, Gaston Barnier et Jean-Rémy Fortoul, auteurs d'ouvrages sur les dialectes de leurs « pays » respectifs : la Drôme et les Alpes du Sud. Ceux qui étaient les interlocuteurs traditionnels de l'ethnologue ne se contentent plus d'offrir des informations brutes aux chercheurs chargés de les organiser ensuite selon une logique qui leur est propre, en fonction des critères, des codes et d'un langage spécialisé pour produire une connaissance au profit d'une communauté scientifique et dans une visée souvent en décalage avec les intérêts de leurs 'informateurs'. Ces chercheurs indigènes décident de présenter leur savoir sur une culture qu'ils considèrent leur appartenir sans la médiation d'un professionnel et de s'adresser d'abord à leur communauté. Le travail de ces passeurs nostalgiques, qui figure dans les bibliothèques des habitants locaux, devient un outil de reconnaissance pour ces passeurs et leurs 'communautés'. Les chercheurs indigènes présentés par Philippe Martel produisent en somme un savoir organique qui parle aux groupes mêmes qui s'identifient dans le dialecte qu'ils étudient (et qu'ils parlent). Cette organicité sociale implique cependant aussi des visées explicites qui mêlent leur engagement à la production de savoir. Des intérêts spécifiques de ces érudits locaux avec lesquels le chercheur extérieur peut ne pas être à l'aise. C'est pour cette raison que lorsque chercheurs diplômés et chercheurs indigènes se rencontrent sur un même terrain, partagent le même sujet et un même but, la production d'un savoir sur celuici, leur relation n'est pas toujours facile, comme on le comprend à la lecture du texte de Sylvie Sagnes. Les chercheurs - et ici en l'occurrence les chercheuses - indigènes ne se reconnaissent pas dans le rôle de «personnes-ressources » que l'ethnologue leur attribue, et revendiquent leur égalité avec les professionnels de la recherche, critiquant le travail des ethnologues lorsque, après leur avoir extrait des informations, ceux-ci les réorganisent dans une perspective que ces chercheuses indigènes ne partagent pas. L'« incompétence » de l'ethnologue, dont le travail est considéré comme " désagréable », se couple en somme au décalage qui existe entre priorités de recherche différentes. Cette impasse se fonde sur un obstacle irréductible : le manque d'engagement reproché par les historiennes locales du costume est revendiqué par la chercheuse universitaire comme condition de sa crédibilité professionnelle.

La transmission engagée des chercheurs indigènes devient militante lorsque les passeurs sont des leaders politiques. Matteo Aria et Adriano Favole présentent des figures emblématiques de passeurs culturels en Océanie dite " francophone ». Jean-Marie Tjibaou en Nouvelle-Calédonie et Duro Raapoto et Henri Hiro en Polynésie-Française sont à l'origine de processus de patrimonialisation partagée qui composent le présent avec l'héritage de la relation coloniale. Qu'ils se construisent dans une démarche non essentialiste (Tjibaou), ou plus centrée sur la revendication d'une diversité irréductible, dans les deux cas il s'agit de célébrer une identité et de se servir du patrimoine de façon instrumentale. Ce caractère militant est encore plus 
marqué lorsque les modalités de la passation deviennent des actions collectives. C'est le cas lorsque le phénomène prend des allures associatives. Guillaume Alévêque décrit l'importance de la composante militante, plutôt que strictement de connaissance, des « reconstructions culturelles » organisées par des associations en Polynésie-Française. Les associations décrites par Véronique Mouliné liées à la mémoire des républicains espagnols refugiés en Languedoc-Roussillon construisent leurs mémoires singulières et sélectives, parfois en conflit avec les historiens, pour affirmer une identité plutôt que pour documenter des événements.

Une autre figure de passeur est celle rencontrée par Magali Demanget dans la Sierra Mazateca, au Mexique et par Arnauld Chandivert dans les Pyrénées : les passeurs sont ici les promoteurs culturels indigènes. Des instituteurs bilingues, qui s'investissent dans l'interprétation du secret chamanique faisant le lien entre les touristes en quête d'authenticité et les chamanes mazatèques, ou entre touristes et organisateurs de fêtes pastorales en relation avec les bergers et avec les agences de développement. La mise en spectacle des cultes de possessions birmans, décrite par Bénédicte Brac de la Perrière, offre un autre exemple de transformation patrimoniale dans laquelle le chercheur, une fois incorporé au groupe de ses interlocuteurs, est investi d'un rôle de passeur de leur culture sur la scène internationale.

Enfin, comme le propose Jean-Louis Tornatore, les passeurs peuvent être multiples, leur expertise découlant non d'une profession patrimoniale mais de la relation à l'objet à patrimonialiser. Nous sommes alors ici face à une « communauté patrimoniale $»^{1}$. Terminé le monopole historien basé sur un savoir érudit qui est resitué sous forme pédagogique au peuple, on assiste à une stratification d'expertises qui dialoguent entre elles, chacune avec ses propres visées. L'expertise anthropologique trouve sa place parmi les autres et, dans ce nouveau contexte, l'auteur propose que l'anthropologue abandonne la neutralité axiologique pour s'engager dans le processus qu'il étudie et assumer la portée éthico-politique du « patrimoine-en-action ».

\section{Postures}

Se revendiquant comme les « témoins, les traductrices et les exégètes » (Ciarcia) de leur communauté d'appartenance, les nouvelles figures d'agents du patrimoine présentées dans ce volume remplacent l'expertise technique et le détachement émotionnel des agents institutionnels du patrimoine (Heinich 2012) par un engagement passionnel. À l'heure où la mise en patrimoine tire sa légitimité de la participation de « porteurs » de patrimoine aux processus de reconnaissance et d'institution du patrimoine, et où l'Unesco demande une institutionnalisation de cette participation dans la fabrique du patrimoine, ce volume présente les effets controversés de cette situation sur les ethnologues ou anthropologues, porteurs d'un savoir et d'une méthodologie scientifique qui étaient jusqu'alors responsables de la validation patrimoniale ou de la production de connaissance.

1 Telle que elle est définie dans la Convention dite de Faro : « personnes qui attachent de la valeur à des aspects spécifiques du patrimoine culturel qu'elles souhaitent, dans le cadre de l'action publique, maintenir et transmettre aux générations futures » (Convention du Conseil de l'Europe sur la valeur du patrimoine culturel pour la société, 2005). 
En effet, si les interlocuteurs de l'ethnologue jouent aujourd'hui le rôle qui avait été auparavant réservé au chercheur, quelle est la place alors dévolue à ce dernier ? C'est une question que, de façon plus ou moins explicite, se posent la plupart des auteurs. Dans les différentes réponses données par les ethnologues qui tentent de négocier un nouveau rôle dans leur interaction avec ces nouveaux agents du patrimoine, la question de l'engagement est posée comme centrale. La proximité entre le chercheur et les acteurs qu'il rencontre sur son terrain et sa participation dans un projet partagé sont saluées par certains (Tornatore) comme une évolution fondamentale pour la réalisation d'une démocratie culturelle, ou sont au contraire présentées comme une dérive hyper-relativiste et déstabilisatrice de l'identité et de l'objectivité scientifique du chercheur (Sagnes).

Si l'ethnologue considère volontiers l'engagement comme un objet d'enquête, il est très mal à l'aise lorsque cet élément se mêle au travail même de recherche (Hastrup et Elsass 1990). Comme le cas présenté par Sylvie Sagnes le montre, l'engagement des acteurs peut être difficile à épouser pour l'ethnologue. Ces mêmes acteurs constituent sans doute un objet d'étude mais est-il possible, ou même souhaitable, pour l'ethnologue de trouver avec eux des voies de collaboration? Non seulement il est difficile pour le chercheur de partager un même langage avec ces acteurs mais quels pourraient être les effets d'un tel effort? Dans le milieu académique, les passeurs d'idées dont parle Nicolas Adell soulèvent la suspicion des collègues. L'imbrication de leur travail avec les objectifs patrimoniaux des acteurs sociaux finirait en fait par légitimer des histoires à soi (Bensa et Fabre 2001) avec ce que Véronique Dassié et Julie Garnier appellent une « onction scientifique ». Est-ce que ces effets font partie des résultats attendus par des chercheurs universitaires ? S'agit-il de comprendre un processus, en l'occurrence celui de la passation du patrimoine, ou d'y participer?

La plupart des auteurs invoquent un passage de la dénonciation critique (invention des traditions) à la compréhension du discours patrimonial des acteurs sociaux. Comment se positionner toutefois vis-à-vis des arguments, des attentes et des intérêts des sujets de la recherche ? L'adoption d'une posture est présentée comme un choix délicat à différents égards. La neutralité est une approche légitime (et légitimante) des sciences sociales qui permet au chercheur de comprendre un objet (Heinich 2002), mais une partie des auteurs de ce volume se demande si la distanciation est la seule façon de pratiquer le métier du chercheur. Véronique Dassié et Julie Garnier parlent d'une proximité «nouvelle » entre chercheur et porteurs de projets patrimoniaux. Dans l'expérience qu'elles présentent, le rôle de l'ethnologue ne tient plus de l'expertise distante, mais de la participation au processus de patrimonialisation comme acteur à part entière. Jean-Louis Tornatore plaide de façon encore plus explicite pour une « posture de proximité » du chercheur qui se doit d'être concerné par les préoccupations des acteurs.

Les négociations de ces nouvelles compétences entre les «porteurs de patrimoine » et les scientifiques que ce volume nous permet d'observer, offrent des réponses à une question centrale, bien formulé par Philippe Martel : « Au fond, à qui revient-il de produire du savoir sur le local ? Faut-il considérer que les mieux armés pour ce faire sont des spécialistes venus du dehors, munis des bons diplômes et des ordres de mission signés par leurs administrations, porteurs du regard détaché qui leur 
permettra de saisir ce qui, pour les indigènes, relève de l'implicite ? " Cette question en suggère une autre, d'ordre plus général : Qu'est-ce que le métier d'anthropologue aujourd'hui ? Si, selon Marc Augé (2006) il y a « encore du pain sur la planche » pour les anthropologues professionnels, les expériences présentées dans ce volume nous montrent l'effort qui est demandé pour négocier une nouvelle posture dans ce contexte. Ces efforts font écho à ceux qui sont déployés bien au-delà de l'anthropologie du patrimoine.

La réflexion des auteurs sur leur posture face aux passeurs de mémoires a le mérite d'entrer en résonance avec les préoccupations les plus actuelles de la discipline et positionne cette façon de faire l'anthropologie du patrimoine comme un chantier expérimental pour l'anthropologie du contemporain, qui, avec la fin des terrains malinowskiens (Faubion et Marcus 2009), est confronté à des nouvelles typologies d'interlocuteurs capables de faire des lectures participantes des textes ethnographiques (Jamin 2005, cité par Ciarcia). Depuis que la distance qui le séparait auparavant de ses « informateurs » a été progressivement supprimée et que les natives talk back (Rosaldo 1986), l'anthropologie a cherché des nouvelles postures pour interagir avec les acteurs sur le terrain. Si cette réflexion a été un sujet fondateur du débat postmoderne dès les années 1980, l'idée d'anthropologie collaborative pousse aujourd'hui le paradigme dialogique à ses conséquences extrêmes. La question de la collaboration avec les interlocuteurs sur le terrain à la fois en tant que moyens et objet de la recherche de terrain s'affirme dès lors comme un débat disciplinaire central.

L'étude des élites a en effet introduit la nécessité de penser au terrain en termes de « complicité » (Marcus 1997) et a amené le besoin de changer les règles du jeu pour intégrer les perspectives et les interprétations des individus ou groupes étudiés dans la définition même du projet et de la méthodologie de recherche. Dans ces cadres « paraethnographiques » (Holmes et Marcus 2008), les anciens « témoins » ou « informateurs » de l'ethnologue sont appréhendés comme des collaborateurs à part entière de la recherche. Mais ces préoccupations ne concernent pas que les anthropologues qui travaillent sur la bureaucratie, la finance, le droit, ou la production scientifique avec des interlocuteurs qui ont souvent un parcours de vie très proche de celui du chercheur (Rabinow 2011). Elles sont partagées dans un courant anthropologique plus vaste qui s'impose dans les assemblées d'anthropologues et prend une forme de plus en plus définie avec un guide qui en résume les enjeux principaux (Lassiter 2005) et une revue ad hoc qui en accueille le débat méthodologique (Collaborative anthropology). L'objectif déclaré d'une telle anthropologie est celui d'instaurer la collaboration avec les acteurs du terrain à toutes les étapes de la recherche à partir de la définition du projet de recherche, et de la prolonger en particulier dans le processus d'écriture, afin de partager la construction du texte ethnographique et les représentations qu'il véhicule avec les groupes étudiés, et d'intégrer les réactions et points de vue émanant de celui-ci dans le texte principal plutôt que de les confiner dans le gris des échanges épistolaires entre l'ethnologue et ses interlocuteurs (Jamin 2005) ou des postfaces aux éditions successives des travaux commentés par les « informateurs ». Tournant indigène délégitimant et hypocrisie populiste (Gross et Plattner 2002) ou, comme le revendiquent ses tenants, évolution nécessaire de la discipline ? Cette controverse, qui traverse l'anthropologie contemporaine, se reflète 
dans la mise en débat aujourd'hui d'une catégorie historiquement aussi centrale pour la discipline que celle d' " informateur ».

Dans l'anthropologie collaborative américaine, l'usage du terme « informateur» a définitivement laissé la place à ceux de « consultant», de « co-intellectuel », voire de « co-chercheur » (Lassiter 2005), ou d' « epistemic partners » (Marcus 2009). Et en Europe, plusieurs chercheurs ont choisi de l'abandonner au profit d'une représentation plus dialogique des échanges de terrain (par ex. Macchiarella 2009). Au même moment toutefois, ces choix, en particulier les expériences d'écriture participative (Hinson 2000), sont considérés comme une stratégie politiquement correcte qui témoigne d'un tournant indigène de la recherche (Martin 2001). La perplexité exprimée par Sylvie Sagnes envers le choix de l'expression « assistantes de recherche » utilisée par une collègue américaine reflète les mêmes préoccupations. Mais à l'exception de cette remarque critique, ce volume ne s'interroge pas sur cette question, qui n'est pas simplement terminologique mais renvoie à l'essence du sujet qu'il analyse. L'usage acritique du terme " informateur », très fréquent dans ce volume, détonne avec son argument principal qui s'attache justement à nous montrer que les passeurs de mémoire ne sont pas simplement des acteurs qui livrent à l'ethnologue des informations sous forme de témoignages. La difficulté de nommer ces nouveaux acteurs dont l'agencéité oblige le chercheur, volens nolens, à un changement de posture reflète le caractère controversé de ce virement participatif non seulement dans la fabrique du patrimoine mais aussi dans la pratique ethnographique.

\section{Références citées}

Adell Nicolas et Yves Pourcher (éds), 2011. Transmettre, quel(s) patrimoine(s) ? Autour du Patrimoine Culturel Immatériel. Paris : Michel Houdiard.

Augé, Marc, 2006. Le Métier d'anthropologue : sens et liberté. Paris : Gallilée.

Bensa Alban et Daniel Fabre (éd.), 2001. Une histoire à soi. Figurations du passé et localité. Paris : MSH.

Berliner, David, 2010. «Anthropologie et transmission », Terrain 55 : 4-19.

Fabre, Daniel, 2002. « Catastrofe, scoperta, intervento o il monumento come evento », in Andreina Ricci (éd.), Archeologia e urbanistica. XII Ciclo di Lezioni sulla Ricerca applicata in Archeologia, Firenze : All'insegna di Giglio : 19-28.

Faubion, James D. et George E. Marcus, 2009. Fieldwork Is Not What It Used to Be : Learning Anthropology's Method in a Time of Transition. Ithaca : Cornell University Press.

Gross, Daniel et Stuart Plattner, 2002. «Anthropology as Social Work. Collaborative Models of Anthropological Research ». Anthropology news 43 (8) : 4.

Hastrup Kirsten \& Peter Elsass 1990. «Anthropological Advocacy : A Contradiction in Terms ? », Current Anthropology 31 (3) : 301-311.

Heinich, Nathalie, 2002. « Pour une neutralité engagée », Questions de communication 2 : 117-127.

-, 2012. « Les émotions patrimoniales : de l'affect a l'axiologie », Social Anthropology 20 : 19-33.

Hinson, Glenn, 2000. Fire in my Bones. Transcendance and the Holy Spirit in African American Gospel. Philadelphia : University of Pennsylvania Press.

Holmes, Douglas R. et George E. Marcus, 2008. « Collaboration Today and the Re-Imagination of the Classic Scene of Fieldwork Encounter », Collaborative Anthropologies 1: 81-101. 


\section{Chiara Bortolotto}

Jamin, Jean, 2005. « Rendez-vous manqué avec le vodou », Gradhiva $1:$ 225-232.

Lassiter, Luke Eric, 2005. The Chicago Guide to Collaborative Ethnography. Chicago : University of Chicago Press.

Le Goff, Jacques (sous la présidence de), 1998. Patrimoine et passions identitaires. Entretiens du Patrimoine 1997. Paris : Fayard.

Macchiarella, Ignazio (éd.) 2009. Cantare a cuncordu. Uno studio a più voci. Udine : Nota.

Marcus, George E., 1997. « The Uses of Complicity in the Changing Mise-en-Scene of Anthropological Fieldwork », Representations, 0, 59:85-108.

-, 2009. «Introduction : notes toward an ethnographic memoir of supervising graduate research through anthropology's decades of transformation », in James D. Faubion and George E. Marcus (éds), Fieldwork is not what it used to be: learning anthropology's method in a time of transition, 1-34. Ithaca, NY : Cornell University Press.

Martin, Denis-Constant, 2001. « Glenn Hinson, Fire in my Bones. Transcendance and the Holy Spirit in African American Gospel », L'Homme 158-159. (En ligne) : [http://lhomme.revues.org/index6414. html.]. Consulté le 20 février 2012.

Rabinow, Paul, 2011. The Accompaniment. Assembling the Contemporary. Chicago and London : University of Chicago press.

Rosaldo, Renato, 1986. When natives talk back: Chicano anthropology since the late sixties, Renato Rosaldo Lecture Series Monograph 2 : 3-20. Tucson : Mexican American Studies and Research Centre. 